J. Clin. Chem. Clin. Biochem.

Vol. 19, 1981, pp. $471-477$

\title{
Zur direkten Bestimmung von Quecksilber im Urin nach der Kaltdampf-Atomabsorptionsspektro- metrie-Methode
}

\author{
Von O. Oster \\ Bioscientia, Institut für Laborintersuchungen, Ingelheim
}

(Eingegangen am 29. August/27. November 1980)

\begin{abstract}
Zusammenfassung: Durch vergleichende Untersuchungen wird belegt, daß sich die Gesamt-Quecksilberkonzentration in Urin $(>2 \mu \mathrm{g} / 1)$ kostensparend direkt durch Atomabsorptionsspektrometrie nach der Kaltdampf-Technik zuverlässig bestimmen läßt, wenn das aus der Urinprobe nach Behandlung mit $\mathrm{KMnO}_{4}$-Lösung und einem $\mathrm{HNO}_{3}-\mathrm{H}_{2} \mathrm{SO}_{4}$-Gemisch mit $\mathrm{NaBH}_{4}$ zu Metalldampf reduzierte Quecksilber abgetrennt und bestimmt wird.
\end{abstract}

Als Vergleichsmethoden dienten die Standardadditionsmethode und ein Verfahren, bei dem die Urinproben noch zusätzlich mit $\mathrm{HNO}_{3}$ in einem PTFE-Druckgefäß mineralisiert wurden. Beide Methoden zeigten, daß die empfohlene Vorschrift das Gesamtquecksilber erfaßt und durch weitere anorganische und organische Harnbestandteile nicht gestört wird. Die Nachweisgrenze der Methode ergab sich zu $2 \mu \mathrm{g} / \mathrm{l}$ Quecksilber (Probenmenge: $1 \mathrm{ml}$ ). Die Variationskoeffizienten lagen bei 7,4\% bzw. 4\% für aufgestockte Hg-Mengen von 10 bzw. $30 \mu \mathrm{g} / \mathrm{l}$. Für nicht exponierte Personen (30 Männer, 30 Frauen) wurde ein „Normal-Wert“ von $<4 \mu \mathrm{g} / 1$ Quecksilber gefunden.

\section{The direct determination of mercury in urine by cold vapor atomic absorption spectroscopy}

Summary: A method is described for the determination of mercury in urine by atomic absorption spectroscopy. The organically bound mercury is oxidized by treatment of the urine with $\mathrm{KMnO}_{4}$, followed by dilution with a mixture of $\mathrm{HNO}_{3}$ and $\mathrm{H}_{2} \mathrm{SO}_{4}$. The sample is then treated with $\mathrm{NaBH}_{4}$ to release the mercury vapor. This procedure measures total mercury (inorganic and organically bound) in the urine. The method was compared with the standard addition method and with the determination after wet digestion with concentrated $\mathrm{HNO}_{3}$. A good correlation was found between the three methods. Other organic and inorganic compounds present in urine do not interfere. Using $1 \mathrm{ml}$ urine samples, the detection limit is $2 \mu \mathrm{g} / 1 \mathrm{Hg}$. Sensitivity is also $2 \mu \mathrm{g} / 1 \mathrm{Hg}$. Urinary mercury in a non-exposed population ( $30 \mathrm{men}, 30$ women) was found to be less than $4 \mu \mathrm{g} / \mathrm{l} \mathrm{Hg}$.

\section{Einfithrung}

Die Bestimmung von Quecksilber in biologischem Material hat auf Grund der Toxizität von Quecksilber und seiner Verbindungen sowie deren Verwendung in Industrie und Landwirtschaft an Bedeutung gewonnen. Die Kontamination der Umwelt in der Bundesrepublik mit quecksilberhaltigen Verbindungen kann durch folgende Überlegungen abgeschätzt werden. Der Gesamtverbrauch an Quecksilber in der Bundesrepublik betrug im Jahre 1971 etwa 660 Tonnen, von denen etwa 300 Tonnen als Verbrauchsprodukte und Abfallstoffe an die Umwelt abgegeben werden (1). Wieviel vion der an die Umwelt abgegebenen Menge schließlich in den menschlichen Organismus gelangt, ist weitestgehend unbekannt. Nach Tölg (2) wird die jährliche anthropogene Belastung der Bundesrepublik durch Quecksilber auf 0,1 mg Quecksilber pro
Quadratmeter Fläche geschätzt. Wenn auch aus diesen Uberlegungen von einer akuten Gefährdung der Bevölkerung durch Quecksilber nicht gesprochen werden kann, ist jedoch eine ständige Überprüfung, inwieweit in Boden, Wasser, Pflanzen- und Tierwelt Quecksilber angehäuft wird, ratsam. Anders ist die Situation bei den in der Quecksilber verarbeitenden Industrie Beschäftigten. Der ständige Kontakt der Beschäftigten mit Quecksilber in der verschiedensten Form macht eine ständige arbeitsmedizinische Überwachung hinsichtlich Quecksilberintoxikationen notwendig. Quecksilber und dessen Verbindungen (anorganische und organische) werden über die Nahrung, die Atmungsorgane und über die Haut in den menschlichen Organismus aufgenommen. Die Uberwachung des mit Quecksilber arbeitenden Personenkreises sollte den Arbeitsplatz (Beach- 
tung der maximalen Arbeitsplatzkonzentration) sowie die Personen selbst einschließen. Aufschluß über Quecksilberintoxikationen sowie deren Grad ergibt neben anderen diagnostischen Kenngrößen vor allem der Quecksilbergehalt des Blutes und des Urins (3).

Der vorliegende Bericht beinhaltet die analytische Bestimmung von Quecksilber im Urin und ist mit folgender Absicht verfaßt worden:

Zur ständigen Uberwachung eines größeren Personenkreises auf Quecksilberintoxikationen sind analytische Methoden notwendig, die schnell und kostengünstig verläßliche Analysenwerte liefern. Eine Methode zur direkten Bestimmung des Quecksilbers im Urin mit Hilfe der Kaltdampf-AAS-Methode und deren routinemäßiger Einsatz im klinisch-chemischen Laboratorium wird beschrieben. Methoden zur Bestimmung des Quecksilbers im Urin sind bereits beschrieben worden; sofern sie mit Hilfe des Quecksilber-Hydrid-Systems erfolgten, konzentrierten sie sich zu sehr auf das Methodische $(4,5)$. Andererseits wurde nie der Beweis geführt, daß die Methoden wirklich das gesamte Quecksilber (anorganisch und organisch gebundenes Quecksilber) erfassen. Ein anderer Mangel besteht darin, daß zu wenige Daten vorliegen, welche die Ergebnisse des Einsatzes des Quecksilber-Hydrid-Systems bei der Bestimmung größerer Probenzahlen beschreiben. Der vorliegende Bericht möchte diese Lücke schließen helfen.

\section{Material und Methoden}

\section{Reagenzien}

Quecksilberstandard (Merck), $\mathrm{KMnO}_{4} \mathrm{Hg}$-arm (Merck), $\mathrm{H}_{2} \mathrm{SO}_{4}$ Hg-arm (Merck), $\mathrm{HNO}_{3} \mathrm{Hg}$-arm (Merck), $\mathrm{NaOH}$ p.a. (Merck), $\mathrm{NaBH}_{4}$ p.a. (Merck), Entschäumer Daw Corning DB 110 A, Methyl-Quecksilber · HCl (Merck), Phenyl-Quecksilber - HCl (Merck), $\mathrm{HNO}_{3}$ suprapur (Merck), $\mathrm{H}_{2} \mathrm{O}$ bidest. aus Milliporeanlage.

Standardreferenz: National Bureau of Standards (NBS) Standard Reference Material 2672, freeze-dried urine certified for mercury.

Entschäumer: Dow Corning DB 110 A Emulsion wird 1:100 verdünnt.

Oxidationslösung: $5 \mathrm{~g} \mathrm{KMnO}_{4}$ auf $100 \mathrm{ml} \mathrm{H}_{2} \mathrm{O}$.

Standardgerade: Es wurde ein Arbeitsstandard hergestellt, der $1 \mathrm{mg} / 1$ in wäßriger Lösung enthält. Aus dem Arbeitsstandard wurde die Standardgerade erstellt, in dem 10, 20,30, 50, 80, $100 \mu l$ des Arbeitsstandards in das Reaktionsgefäß gegeben wurden. Die pipettierte Menge entspricht 10, 20, 30, 50, 80, $100 \mathrm{ng} \mathrm{Hg}$ absolut. Die Bestimmungen für die Standardgerade wurden unter identischen Bedingungen wie im Reaktionsansatz für die direkte Bestimmung von Quecksilber im Urin durchgeführt.

Reaktionsansatz für die direkte Bestimmung von Quecksilber im Urin: $1 \mathrm{ml}$ Urin wird in das Reaktionsgefäß vorgelegt. $\mathrm{Zu}$ dem Urin werden $0,2 \mathrm{ml} \mathrm{KMnO}$-Lösung pipettiert und gut gemischt. Anschließend werden $10 \mathrm{ml}$ Säuremischung in das Reaktionsgefäß gegeben und $1 \mathrm{ml}$ Entschäumerlösung hinzugefügt. Es wurden jeweils Doppelbestimmungen durchgeführt.

Reaktionsansatz fuir die Standardadditionsmethode: Der Reaktionsansatz für die Standardadditionsmethode entspricht dem Reaktionsansatz für die direkte Bestimmung von Quecksilber im Urin. Es wurden immer mindestens 3 Zugaben getätigt: in der Regel 10, 30 und $50 \mathrm{ng}$ absolut.

Reaktionsansatz nach Aufschluß der Urinprobe: Nach dem Aufschluß wurde der Urin mit der Säuremischung in das Reaktionsgefäß quantitativ übergeführt und wie bei der direkten Bestimmung gemessen. Verluste wurden durch Koritrollen ausgeschaltet.

Aufschluß: Der Aufschluß wurde in der Tölg-Bombe mit $\mathrm{HNO}_{3}$ suprapur durchgeführt. $1 \mathrm{ml}$ Urin und $1 \mathrm{ml} \mathrm{HNO}_{3}$ konz. wurden unter Druck 3 Stunden bei $100^{\circ} \mathrm{C}$ aufgeschlossen.

Interferenzstudie: Zur Untersuchung von Interferenzen wurde Urin auf $30 \mu \mathrm{g} / \mathrm{l}$ aufgestockt und die entsprechenden Reagenzien in den in Tabelle 3 angegebenen Konzentrationen hinzugefügt.

Normalwertstudie: die Urine wurden in $50 \mathrm{ml}$ Polyethylenflaschen gesammelt und unmittelbar anschließend mit $0,5 \mathrm{ml}$ $\mathrm{HNO}_{3}$ konz. angesäuert und gemessen. War eine Lagerung der Urine notwendig, wurden die Proben bei $-20^{\circ} \mathrm{C}$ gelagert. Beim Auftauen der Urine wurden keine Verluste festgestellt. Die Probengefäße gaben weniger als 0,5 ng Quecksilber $a b$.

Apparate: Die Reduktion des Quecksilbers wurde mit dem Mercury-Hydride-System MHS 20 der Firma Perkin Elmer durchgeführt. Bevor die Reaktion durch Hinzugabe von $\mathrm{NaBH}_{4}$ Lösung in Gang gesetzt wurde, erfolgte eine Spülung des Reaktionsgefäßes mit Argon (9 s). Die Reaktionszeit wurde so gewählt, daß innerhalb der Reaktionszeit das Peakmaximum überschritten wurde $(9 \mathrm{~s})$. Die Küvette wurde auf $200^{\circ} \mathrm{C}$ aufgeheizt. Gemessen wurde mit dem Atomabsorptionsspektrometer AAS 5000 der Firma Perkin Elmer; Wellenlänge $253,6 \mathrm{~nm}$. Es wurde eine elektrodenlose Lampe als Strahlungspunkt verwendet.

\section{Ergebnisse und Diskussion}

Eine Reihe von Methoden wurden beschrieben, die den hohen Dampfdruck von metallischem Quecksilber ausnutzen, um quantitativ Quecksilber zu bestimmen $(6,7$, 8). Anorganische Quecksilberverbindungen müssen daher in elementares Quecksilber übergeführt werden, was durch ein entsprechendes Reduktionsmittel, meistens $\mathrm{SnCl}_{2}$ oder $\mathrm{NaBH}_{4}$ geschieht. Bei der Bestimmung von Quecksilber in biologischem Material z. B. den Körperflüssigkeiten Blut und Urin liegt Quecksilber jedoch nicht nur in der anorganischen Form vor, sondern auch organisch gebunden, und zwar in der Regel als Methylquecksilber: Magos (9) berichtet, daß im Blut $66 \%$ des Gesamtquecksilbers organisch gebunden, meistens als Methylquecksilber, vorliegen. Urinanalysen auf Quecksilber sollten, will man das Ausmaß einer Intoxikation richtig abschätzen können, den Gesamtgehalt an Quecksilber bestimmen. Toffaletti et al. (4) beschrieben eine Methode zur Quecksilberbestimmung im Urin, die, wie die hier beschriebene Methoden, $\mathrm{NaBH}_{4}$ als Reduktionsmittel verwendet. Der Urin wird ohne Vorbehandlung eingesetzt und die Bestimmung des Gesamtquecksilbers dadurch erreicht, daß der entstehende Quecksilberdampf in eine auf $850^{\circ} \mathrm{C}$ vorgeheizte Quarzküvette geleitet wird, wo eine Zersetzung der organischen Quecksilberverbindungen erfolgt und eine simultane Bestimmung des anorganischen und organisch gebundenen Quecksilbers erlaubt. Die hohe Atomisierungstemperatur der Küvette bedingt jedoch eine Herabsetzung der Empfindlichkeit. Die hier beschriebene Methode nimmt eine Vorbehandlung der Urinprobe vor, durch die organisch 
gebundenes Quecksilber mit $\mathrm{KMnO}_{4}$ im sauren Medium zu zweiwertigem Quecksilber oxidiert wird (Reaktionszeit etwa 1 Minute). Nach der Oxidation wird das zweiwertige Quecksilber mit einem Úberschuß an $\mathrm{NaBH}_{4}$, das im sauren Medium einen Uberschuß Wasserstoff liefert, zu elementarem Quecksilber reduziert. Mit einem Argonstrom wird das elementare Quecksilber in eine auf $200^{\circ} \mathrm{C}$ vorgeheizte Quarzküvette geleitet, wo das Quecksilber atomabsorptionsspektrometrisch bestimmt wird. Eine Heizung der Küvette ist nicht unbedingt notwendig, verhindert jedoch die Kondensation von Wasserdampf in der Küvette.

Um den Beweis zu führen, daß mit der direkten Bestimmung von Quecksilber im Urin nach dessen Behandlung mit $\mathrm{KMnO}_{4}$ im sauren Medium das gesamte Quecksilber ermittelt wird, wurde die direkte Bestimmung mit der Bestimmung nach der Standardadditionsmethode und der Bestimmung nach vorhergegangenem Aufschluß mit $\mathrm{HNO}_{3}$ verglichen. Die Quecksilberkonzentration in 22 Urinproben (in Tab. 1 sind die Ergebnisse zahlenmäßig zusammengefaßt) wurde

1. direkt im Urin bestimmt und nach einer Standardgeraden aus wäßrigen Standards ausgewertet;

2. nach der Standardadditionsmethode bestimmt;

3. nach einem Aufschluß in der Tölg-Bombe bestimmt.

In den Abbildungen 1-3 sind die Ergebnisse der Regressionsanalysen graphisch dargestellt. Auf der Abscisse ist die jeweilige Bezugsmethode aufgetragen. Es wurden die Ergebnisse aus der direkten Bestimmung (Abb. 1) und der Standardadditionsmethode (Abb. 2) mit den Ergeb-

Tab. 1. Vergleich der Ėrgebnisse der direkten Bestimmung von Quecksilber im Ưrin mit der Bestimmung nach Aufschluß sowie der Standardadditionsmethode; Werte in $\mu \mathrm{g} / 1$ Urin.

\begin{tabular}{lccc}
\hline Lfd. Nr. & $\begin{array}{l}\text { Direkte } \\
\text { Bestimmung }\end{array}$ & $\begin{array}{l}\text { Standard- } \\
\text { addition }\end{array}$ & Aufschluß \\
\hline 1 & 12 & 11 & 10 \\
2 & 46 & 48 & 51 \\
3 & 41 & 46 & 40 \\
4 & $<2$ & $<2$ & $<2$ \\
5 & $<2$ & $<2$ & $<2$ \\
6 & $<2$ & $<2$ & 2 \\
7 & 313 & 320 & 331 \\
8 & 335 & 305 & 312 \\
9 & 148 & 144 & 139 \\
10 & 2 & 2 & $<2$ \\
11 & 5 & 6 & 7 \\
12 & 7 & 6 & 8 \\
13 & 85 & 88 & 79 \\
14 & $<2$ & $<2$ & $<2$ \\
15 & 6 & 6 & 7 \\
16 & 93 & 90 & 85 \\
17 & 121 & 117 & 112 \\
18 & 174 & 172 & 181 \\
19 & 223 & 228 & 237 \\
20 & 291 & 281 & 265 \\
21 & 62 & 56 & 68 \\
22 & 76 & 82 & 73 \\
\hline
\end{tabular}

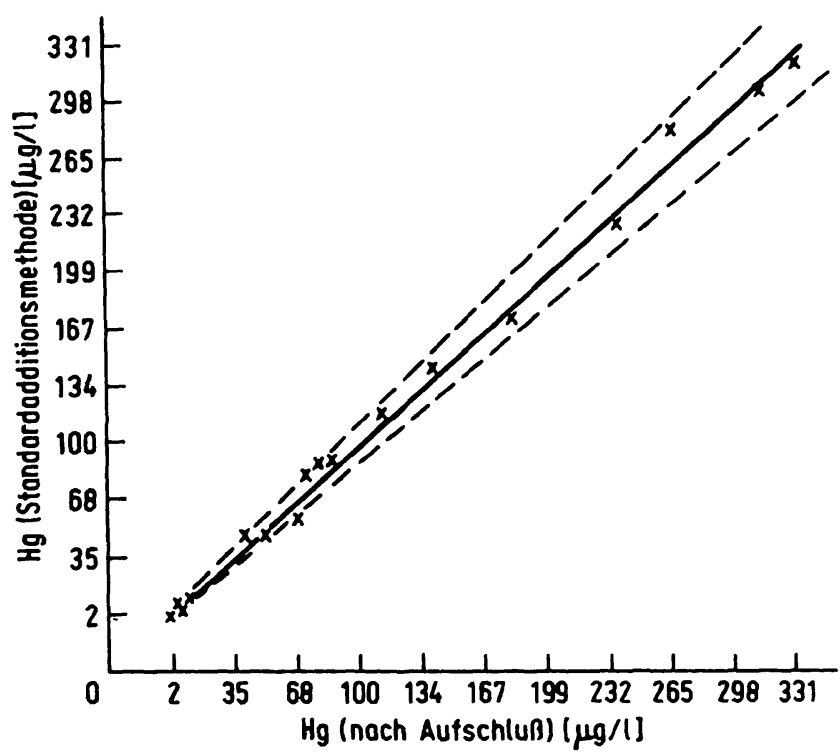

Abb. 1. Korrelation der direkten Quecksilber-Bestimmung mit der Bestimmung nach Aufschluß (Bezugsmethode). Auf der Ordinate und der Abszisse sind die Werte in $[\mu \mathrm{g} / \mathrm{l}] \mathrm{Hg}$ aufgetragen. Die unterbrochene Linie gibt den 10\%-Abweichungsbereich an $y=0,17+1,01 x ; r=1,00$.

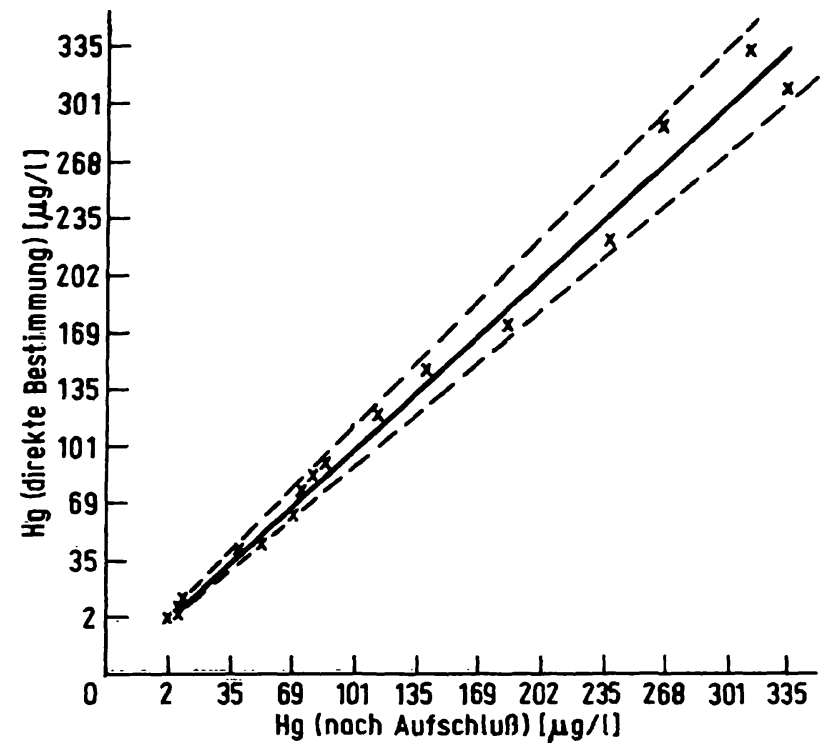

Abb. 2. Korrelation der Standardadditionsmethode mit der Quecksilber-Bestimmung nach Aufschluß (Bezugsmethode). Auf der Ordinate und der Abszisse sind die Werte in $[\mu \mathrm{g} / 1] \mathrm{Hg}$ aufgetragen. Die unterbrochene Linie gibt den $10 \%$-Abweichungsbereich an. $y=1,23+0.98 x ; r=1,00$.

nissen aus der Bestimmung des Quecksilbers nach Aufschluß mit $\mathrm{HNO}_{3}$ korreliert. Ferner wurden die Ergebnisse aus der direkten Bestimmung mit den Ergebnissen der Standardadditionsmethode korreliert (Abb. 3). Die Korrelationskoeffizienten sowie die Steigung der Geraden und die Achsenabschnitte sowie die Werte für den t- und f-Test sind in Tabelle 2 gegenübergestellt. Es ist eine gute 


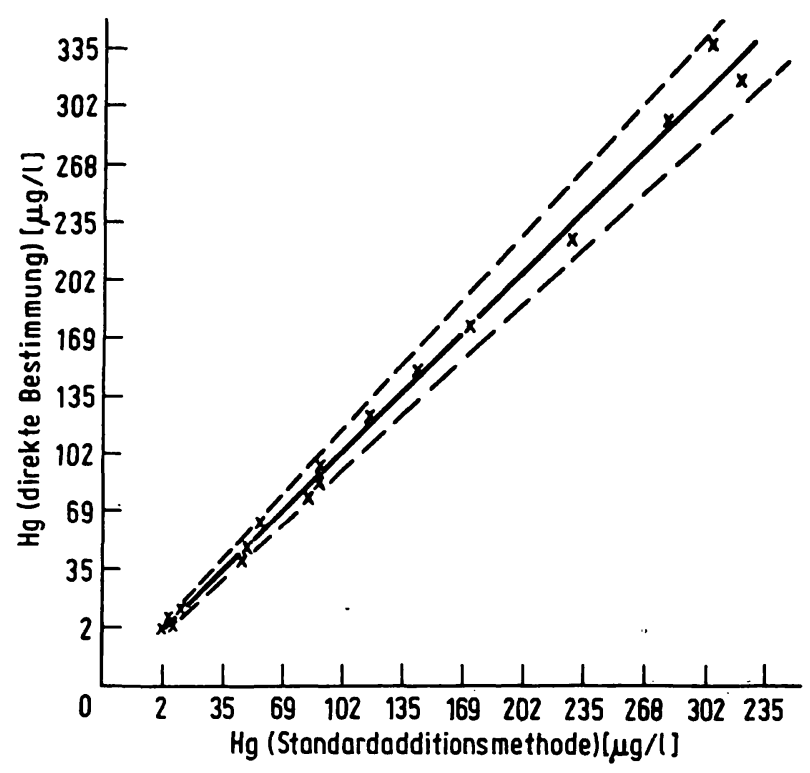

Abb. 3. Korrelation der direkten Quecksilber-Bestimmung mit der Standardadditionsmethode (Bezugsmethode). Auf der Ordinate und der Abszisse sind die Werte in $[\mu \mathrm{g} / \mathrm{l}] \mathrm{Hg}$ aufgetragen. Die unterbrochene Linie gibt den $10 \%$ Abweichungsbereich an. $y=1,09+1,03 x ; r=1,00$.
Korrelation der Ergebnisse festzustellen. Verglichen wurden die Methoden, bei denen nach Peakhöhe und ohne Untergrundkompensation gearbeitet wurde. Die Bestimmung von Quecksilber nach Aufschluß wurde als Bezugsmethode verwendet (obwohl sie den höchsten Variationskoeffizienten zeigt), weil beim Aufschluß das gesamte organisch gebundene Quecksilber in anorganisches zweiwertiges Quecksilber übergeführt wird. Die gute Übereinstimmung der Methoden bestätigt, daß die direkte Bestimmung die Gesamtkonzentration (anorganisch und organisch gebundenes Quecksilber) des Quecksilbers im Urin erfaßt. Die Oxidation der organischen Quecksilberverbindungen mit $\mathrm{KMnO}_{4}$ im sauren Medium ist somit vollständig. Versuche mit Urin, dem organische Quecksilberverbindungen zugesetzt wurden (Methylquecksilberchlorid und Phenylquecksilberchlorid) bestätigen, daß bei der direkten Bestimmung von Quecksilber im Urin nach Oxidation mit $\mathrm{KMnO}_{4}$ das gesamte Quecksilber erfaßt wird. In Tabelle 3 sind die Ergebnisse der Bestimmung von zugesetzten organischen Quecksilberverbindungen zusammengefaßt. Die Wiederfindungsrate ist $100 \%$. Der Vergleich der drei verschiedenen Methoden bestätigt auch die a priori gemachte Annahme,

Tab. 2. Zusammenfassung der Korrelationskoeffizienten, der Steigung und der Achsenabschnitte der Korrelationsgeraden sowie der statistischen Parameter der $\mathrm{t}$ - und der $\mathrm{f}$-Statistik. Verglichen wurden die direkte Bestimmung und die Standardadditionsmethode mit dẹ Bestimmung nach Aufschluß und die direkte Bestimmung mit der Standardadditionsmethode.

\begin{tabular}{llllllll}
\hline Bezugsmethode & Vergleichsmethode & $\begin{array}{l}\text { Korre- } \\
\text { lations- } \\
\text { koeffizient }\end{array}$ & $\begin{array}{l}\text { Korrelations- } \\
\text { gerade } \\
\mathrm{y=a}+\mathrm{bx} \\
\mathrm{a}\end{array}$ & $\mathrm{b}$ & t-Statistik & f-Statistik & $\begin{array}{l}\text { Freiheits- } \\
\text { gerade }\end{array}$ \\
\hline Aufschluß & Direkte Bestimmung & 0,996 & 0,17 & 1,01 & 0,709 & 1,04 & 21 \\
Aufschluß & Standardaddition & 0,997 & 1,23 & 0,99 & 0,031 & 1,02 & 21 \\
Standardaddition & Direkte Bestimmung & 0,998 & $-1,09$ & 1,03 & 0,908 & 1,06 & 21 \\
\hline
\end{tabular}

Tab. 3. Zusammenfassung der Ergebnisse eines auf $30 \mu \mathrm{g} / 1 \mathrm{mit}$ den organischen Quecksilberverbindungen MethylQuecksilber $\cdot \mathrm{HCl}$ und Phenyl-Quecksilber $\cdot \mathrm{HCl}$ aufgestockten Urins. Verglichen wurde die Hg-Bestimmung ohne und mit $\mathrm{KMnO}_{4}$-Zugabe.

\begin{tabular}{llll}
\hline Verbindung & $\begin{array}{l}\mathrm{Hg}-\text { Konzen- } \\
\text { tration des } \\
\text { aufgestockten } \\
\text { Urins } \\
{[\mu \mathrm{g} / \mathrm{l}]}\end{array}$ & $\begin{array}{l}\text { Wiederfindung } \\
\text { ohne } \mathrm{KMnO}_{4}^{-} \\
\text {Zugabe }\end{array}$ & $\begin{array}{l}\text { Wiederfindung } \\
\text { mit } \mathrm{KMnO}_{4}- \\
\text { Zugabe }\end{array}$ \\
\hline$\mu \mathrm{g} / 1]$ & {$[\mu \mathrm{g} / \mathrm{l}]$} \\
\hline Methyl- & 30 & 3 & 31 \\
Queck- & 30 & 4 & 32 \\
silber- $\mathrm{HCl}$ & 30 & 5 & 31 \\
& 30 & 4 & 33 \\
& 30 & 4 & 31 \\
Phenyl- & 30,6 & 2 & 32 \\
Queck- & 30,6 & 4 & 32 \\
silber- $\mathrm{HCl}$ & 30,6 & 3 & 33 \\
& 30,6 & 3 & 34 \\
& 30,6 & 4 & 32 \\
\hline
\end{tabular}

daß die Additionsmethode zumindest in diesem Falle als Referenżmethode verwendet werden kannn. Eine weitere Bestätigung liefert die Parallelität der Geraden, ermittelt über die Standardadditionsmethode mit der Standardgeraden aus wäßrigen Standards, die aus Abbildung 4 hervorgeht. Der Vorteil des Hydridsystems, die fast vollständige Abtrennung der Matrix, ist sicher für diese gute Übereinstimmung mitverantwortlich. Inwieweit die Matrix Einfluß auf das direkte Bestimmungsverfahren hat, wurde durch Zugaben von möglichen Urinbestandteilen zu einem auf $30 \mu \mathrm{g} / \mathrm{l}$ mit Quecksilber aufgestockten Urin untersucht. Dabei . wurde auch berücksichtigt, ob reduzierende organische Substanzen die $\mathrm{KMnO}_{4}$-Oxidation beeinflussen können. Wie aus Tabelle $4 \mathrm{zu}$ ersehen ist, wurden keine Inter= ferenzen möglicher Harnbestandteile bis zu-den in Tabelle 4 angegebenen Konzentrationen festgestellt. 


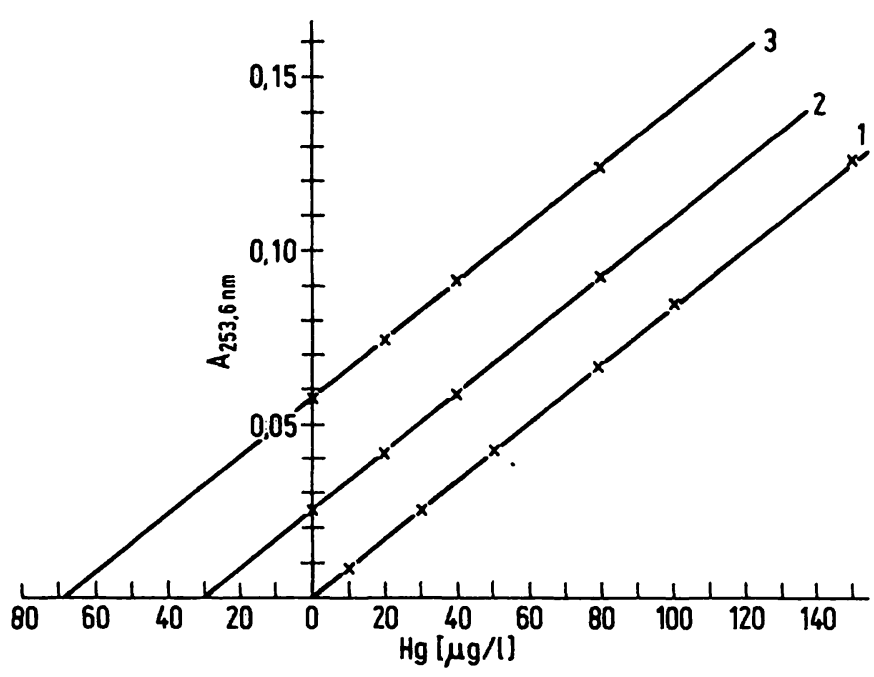

Abb. 4. Graphische Darstellung der Standardgeraden (1) sowic der Geraden nach der Standardaddition für einen auf $30 \mu \mathrm{g} / \mathrm{Hg}$ aufgestockten Urin (2) und einen Urin unbekannter Quecksilber-Konzentration (3).

Um die geeignetste Bestimmungsmethode zu ermitteln, wurde die Präzision der einzelnen Bestimmungsverfahren unter den verschiedensten Bedingungen verglichen. Verglichen wurde (Tab. 5) wiederum die direkte Bestimmungsmethode mit der Standardadditionsmethode sowie der Bestimmung von Quecksilber nach Aufschluß. Während die direkte Bestimmungsmethode und die Standardadditionsmethode vergleichbare Variationskoeffizienten aufweisen, wird ein etwas höherer Varia-
Tab. 4. Zusammenfassung der Ergebnisse der Untersuchung möglicher Interferenzen von im Urin vorkommenden Substanzen auf die direkte Bestimmung von Quecksilber im Urin, bezogen auf einen auf $30 \mu \mathrm{g} / \mathrm{l}$ aufgestockten Urin.

\begin{tabular}{llll}
\hline $\begin{array}{l}\text { Untersuchte } \\
\text { Interferenz }\end{array}$ & $\begin{array}{l}\text { Konzentration } \\
\text { der inter- } \\
\text { ferierenden } \\
\text { Substanz }\end{array}$ & Hg gefunden & $\begin{array}{l}\text { Wiederfindungs- } \\
\text { rate für } \mathrm{Hg}\end{array}$ \\
\hline Glucose & $50 \mathrm{mg} / \mathrm{l}]$ & 31 & {$[\%]$} \\
$\begin{array}{l}\text { Ascorbin- } \\
\quad \text { säure }\end{array}$ & $50 \mathrm{mg} / \mathrm{l}$ & 31 & 100 \\
Cystein & $10 \mathrm{mg} / \mathrm{l}$ & 32 & 100 \\
Arsen & $100 \mu \mathrm{g} / \mathrm{l}$ & 30 & 100 \\
Blei & $100 \mu \mathrm{g} / 1$ & 31 & 100 \\
Kupfer & $100 \mu \mathrm{g} / 1$ & 31 & 100 \\
Selen & $100 \mu \mathrm{g} / 1$ & 30 & 100 \\
Thallium & $100 \mu \mathrm{g} / 1$ & 30 & 100 \\
\hline
\end{tabular}

tionskoeffizient gefunden, wenn Quecksilber nach dem Aufschluß bestimmt wird. Dieses Ergebnis ist nicht überraschend, da beim Aufschluß Verluste sowie Kontaminationen, verglichen mit den direkten Bestimmungsmethoden, vermehrt eintreten. Gegenübergestellt wurden die Bestimmungsmethoden, bei denen nach Peakhöhe ausgewertet und ohne Untergrundkompensation gemessen wurden. Eine Untergrundkompensation hat sich als nicht notwendig erwiesen und, da mit Untergrundkompensation ein höherer Variationskoeffizient gefunden wurde, auf diese verzichtet. Ebenso wurde für die Auswertung der direkten Bestimmungsmethode mittels Peakfläche ein höherer Variationskoeffizient gefunden.

Tab. 5. Zusammenfassung der für die einzelnen Bestimmungsmethoden (dịrekte Bestimmung, Aufschluß, Standardaddition) gefundenen Variationskoeffizienten (VK); bei der Ermittlung der Variationskoeffizienten wurden mindestens 10, in der Regel 15 Bestimmungen durchgefuihrt.

\begin{tabular}{llllll}
\hline Bestimmungsmethode & $\begin{array}{l}\text { Auf } 10 \mu \mathrm{g} / 1 \mathrm{Hg} \\
\text { aufgestockter } \\
\text { Urin }\end{array}$ & $\begin{array}{l}\text { Auf } 30 \mu \mathrm{g} / 1 \mathrm{Hg} \\
\text { aufgestockter }\end{array}$ & $\begin{array}{l}\text { NBS-Low Level } \\
\text { Urine (1) }\end{array}$ & $\begin{array}{l}\text { NBS-High Level } \\
\text { Urine (2) }\end{array}$ & $\begin{array}{l}\text { VK von Tag } \\
\text { zu Tag } \\
\text { (mind. 10 Tage) } \\
\end{array}$ \\
& & & & {$[\%]$} & {$[\%$}
\end{tabular}

Direkte Bestimmung:

a) Peakhöhenauswerntung ohne Untergrundkompensation

b) Peakhöhenauswertung mit Untergrundkompensation

c) Peakflächenauswertung ohne Untergrundkompensation

Aufschluß; Peakhöhenauswertung ohne Untergrundkompensation

Standardaddition; Peakhöhenauswertung ohne Untergrundkompensation

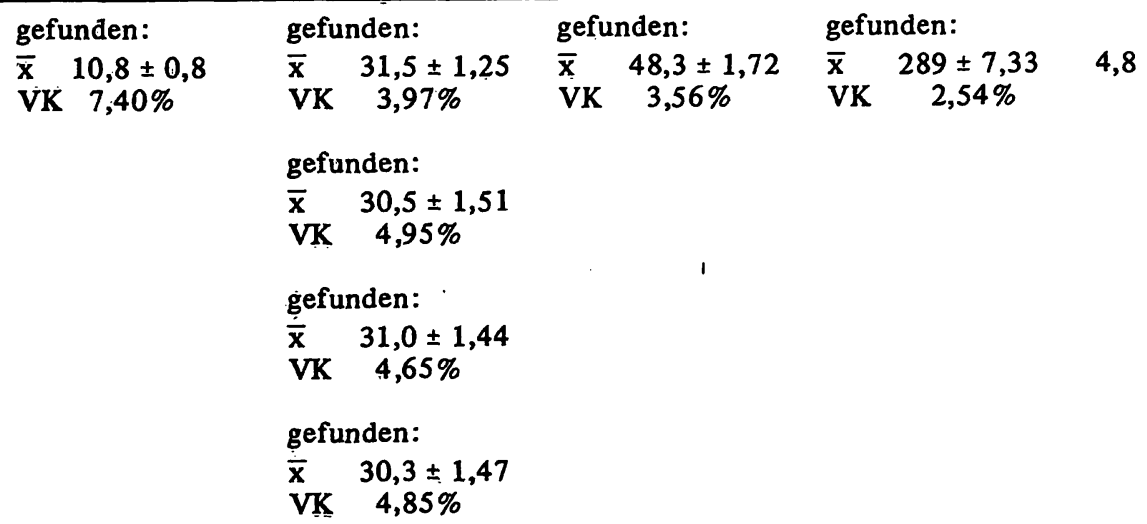

(1) NBS-Spezifikation 49,8 $\pm 4,2 \mu \mathrm{g} / \mathrm{l}$; (2) NBS-Spezifikation $294 \pm 24 \mu \mathrm{g} / \mathrm{l}$. 
Geringe Veränderungen der Basislinie wirken sich beim Auswerten nach Peakfläche stärker aus als beim Auswerten nach der Peakhöhe. Bessere Ergebnisse mittels der Peakhöhe als mit der Peakfläche werden aber nur dann erzielt, wenn die Gaszufuhr konstant gehalten wird. Die Auswertung nach Peakhöhe hat den Vorteil, daß sie auch mit Geräten durchzuführen ist, die keine Integrationsmöglichkeit besitzen und nur mit Hilfe eines Schreibers auswerten. Die in dieser Arbeit mitgeteilten Ergebnisse wurden ohne Untergrundkompensation gemessen und nach Peakhöhe ausgewertet. Die Nachweisgrenze wird mit $2 \mathrm{ng} \mathrm{Hg}$ absolut oder $2 \mu \mathrm{g} / \mathrm{l}$, wenn $1 \mathrm{ml}$ Urin zum Einsatz kommt, angegeben. Als Nachweisgrenzenkriterium dient einerseits das Zweifache der Standardabweichung, bestimmt bei einem Wert, der möglichst nahe der Nachweisgrenze liegt, anderseits das Zweifache des Mittelwerts des Rauschens der Basislinie. Für beide Kriterien wurden als Nachweisgrenze 2 ng absolut ermittelt. Die Empfindlichkeit des Nachweises mit der Atomabsorption, definiert als die Menge, die eine Absorption von $1 \%$ ergibt, wird ebenfalls mit 2 ng absolut angegeben. Die Nachweisgrenze der Bestimmung von Quecksilber im Urin kann gesenkt werden, wenn eine größere Menge Urin eingesetzt wird. Es ist jedoch darauf hinzuweisen, daß die Kontrolle der Kontaminationen, wenn kleinere Mengen als $1 \mu \mathrm{g} / 1$ nachgewiesen werden sollen, oft sehr schwierig und aufwendig ist (10).

Mit der direkten Bestimmungsmethode wurde der Urin von 60 Personen (30 Männer und 30 Frauen im Alter zwischen 20 und $40 \mathrm{Jahren}$ ), die nicht mit quecksilberhaltigen Substanzen regelmäßig in Berührung kommen, untersucht. In Abbildung 5 ist ein Histogramm dargestellt, das die Ergebnisse der Untersuchung zusammenfaßt. Danach liegen die Quecksilberkonzentrationen des Urins von über $95 \%$ aller untersuchten Personen unter $4 \mu \mathrm{g} / \mathrm{l}$ Urin. Wie man aus dem Histogramm entnehmen

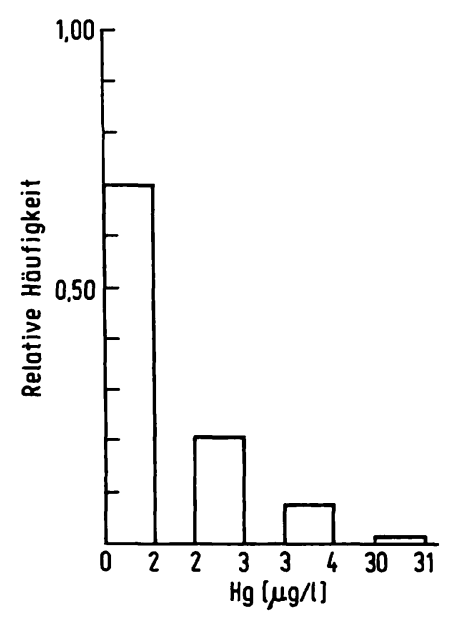

Abb. 5. Histogramm der Verteilung der Quecksilberkonzentration im Urin des untersuchten Personenkreises aus dem Raum Mainz, Ingelheim, Bingen, Bad Kreuznach. kann, erlaubt die direkte Bestimmungsmethode mit der Nachweisgrenze von 2 ng absolut durchaus Messungen im Bereich der Urin-Quecksilberkonzentration nicht exponierter Personen. Ein Unterschied der Quecksilberkonzentrationen im Urin zwischen Männern und Frauen kann nicht angegeben werden, da die Bestimmungsmethode nur den oberen Bereich der Gauss-Verteilung erfaßt. Das Ergebnis, daß bei einem nicht exponierten Personenkreis weniger als $4 \mu \mathrm{g} / 1$ Urin gefunden wird, stimmt mit den mit der Atomabsorption ermittelten Werten von Kubasik et al. (11) $(1,4 \mu \mathrm{g} / 1$ Urin) und mit denen von Bostroem et al. (12) $(2,7 \mu \mathrm{g} / 1) \mathrm{mit}$ der Atomemmissionsspektroskopie ermittelten Werten gut überein. Ebenso gute Übereinstimmung ist vorhanden mit Quecksilberkonzentrationen im Urin, die mit der Neutronenaktivierungsanalyse $(13,14)$ gefunden wurden ( $1 \mu \mathrm{g} / 1$ und 2,9 $\mu \mathrm{g} / 1$ Urin). Die gute Übereinstimmung der in dieser Arbeit mit dem Hydrid-System ermittelten Quecksilberkonzentration mit Werten der Neutronenaktivierungsanalyse, bei der die Probe ohne jegliche Vorbehandlung direkt gemessen wird, zeigt, daß analytische Verfahren, die auf verschiedenen physikalischen Eigenschaften eines Stoffes beruhen, durchaus zu vereinbaren Werten kommen können, wenn Kontaminationsmöglichkeiten und Verluste nahezu eingeschränkt werden. Direkte Bestimmungsverfạhren sind sicher ein Meilenstein auf dem Wege, Kontaminationsmöglichkeiten und Verluste zu kontrollieren.

Die untersuchte Personengruppe stammt aus dem Gebiet Mainz, Ingelheim, Bingen, Bad Kreuznach. Es ist nicht auszuschließen, daß Bevölkerungsgruppen in meeresnahen Gegenden, deren Meeresfischkonsum einen höheren Stellenwert in der täglichen Nahrung aufweist, mehr Quecksilber als $4 \mu \mathrm{g} / 1$ Urin ausscheiden $(15,16)$. Ebenso ist bei Populationen, die in der Nähe quecksilberhaltige Stoffe verarbeitender Industrien wohnen oder in diesen arbeiten, ein höherer Quecksilbergehalt im Urin zu erwarten.

In die Untersuchungen miteinbezogen wurden 88 Personen, bei denen Verdacht bestand, daß ihre Gesundheit durch Quecksilber und dessen Verbindungen geschädigt sein oder werden könnte. In Abbildung 6 sind die Ergebnisse in einem Histogramm zusammengefaßt. Ǔber $42 \%$ der untersuchten Urine zeigen höhere Quecksilberkonzentrationen als das untersuchte Bezugskollektiv $(<4 \mu \mathrm{g} /$ 1) aus dem Raum Mainz, Ingelheim, Bingen, Bad Kreuznach. In über $24 \%$ der untersuchten Urine wurden höhere Quecksilberkonzentrationen als $10 \mu \mathrm{g} / \mathrm{l}$ gefunden; in fast $16 \%$ der Urine wurden Quecksilberkonzentrationen von mehr als $20 \mu \mathrm{g} / \mathrm{l}$ gefunden.

\section{Schlußfolgerungen}

Die vorliegende Untersuchung hat den Beweis gefuhrt, daß bei der direkten Bestimmung des Quecksilbers im Urin nach $\mathrm{KMnO}_{4}$-Oxidation im sauren Medium mit 


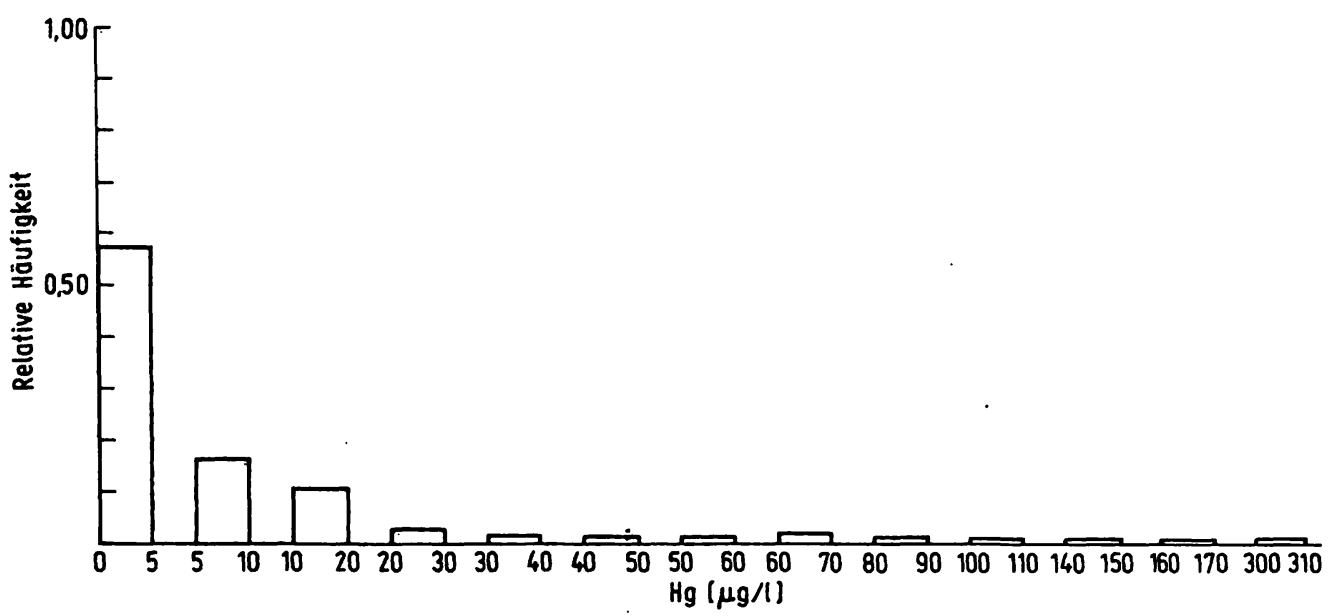

Abb. Histogramm, die Verteilung der Hg-Werte im Urin einer Personengruppe mit Verdacht auf Hg-Intoxikation darstellend.

der $\mathrm{NaBH}_{4}$-Reduktion das gesamte (anorganisch und organisch gebundene) Quecksilber erfaßt wird. Es ist somit möglich, mit einer schnellen, kostengünstigen Messung im Konzentrationsbereich zu messen, der dem Normalwertbereich von Quecksilber im Urin entspricht. Der Zeitaufwand (etwas mehr als zwei Minuten pro Messung inklusive Probenvorbereitung) liegt bei einer Doppelbestimmung bei etwa 5 Minuten und somit innerhalb vertretbarer Grenzen. Interferenzen, soweit sie bisher abschätzbar sind, traten bei der direkten Bestimmung von Quecksilber im Urin nicht auf. Der Urin-Quecksilbergehalt eines aus dem Gebiet Mainz, Ingelheim, Bịgen,
Bad Kreuznach stammenden Personenkreises nicht quecksilberbelasteter Personen wurde mit weniger als $4 \mu \mathrm{g} / 1$ Urin ermittelt. In den berufsgenossenschaftlichen Grundsätzen für arbeitsmedizinische Vorsorgeuntersuchungen (17) wird ein Normalwertbereich angegeben, der unter $20 \mu \mathrm{g} / 1$ Urin beträgt. Der in der vorliegenden Studie gefundene Wert von weniger als $4 \mu \mathrm{g} / 1$ liegt deutlich unter dem des in den berufsgenossenschaftlichen Grundsätzen zitierten Wertes von weniger als $20 \mu \mathrm{g} / \mathrm{l}$, aber in einer durchaus vergleichbaren Größenordnung mit dem von Schaller et al. (18) berichteten Normalwert von 4,31 $\pm 2,33 \mu \mathrm{g} / \mathrm{l}$.

\section{Literatur}

1. Dér Rat von Sachverständigen für Umweltfragen, Umweltgutachten 1978, S. 55, W. Kohlhammer, Stuttgart und Mainz.

2. Tölg, G. (1975), Erzmetall 390-395.

3. Report of an International Commitee (1969), Arch. Environ Health 19, 891-905.

4. Toffaletti, J. \& Savory, J. (1975), Anal. Chem. 47, 2091-2095.

5. Lindstedt, G. (1970), Analyst 95, 264-271.

6. Magos, L. (1971), Analyst 96, 847-853.

7. Melicher, M. (1978), Perkin Elmer Lab Notes 23.

8. Abo-Rady, M. D. K. (1979), Z. Anal. Chem. 299, 187-189.

9. Magos, L. (1972), J. Am. Organization Anal. Chem. 55, 966-971.

10. Kaiser, G., Götz, D., Knapp, B., Maichin, B., Spitzy, H. \& Tölg, G. (1978), Z. Anal. Chem. 291, 278-291.
11. Kubasik, N. P., Sine, H. E. \& Volosin, M. T. (1972), Clin. Chem. 18, 1326-1329.

12. Bostroem, H. \& Wester, O. (1969), Acta Endocrinol. Kbh. $60,380-383$.

13. Wester, P. O. (1973), Acta Med. Scand. 194, 505-507.

14. Bostroem, H. \& Wester, P. (1968), Acta Med. Scand. 183, 209-212.

15. Gardner, D. (1978), Nature 272, 49-51.

16. Dennis, C. A. R. \& Fehr, F. (1975), Science Total Environ. 3, 267-274.

17. Berufsgenossenschaftliche Grundsätze für arbeitsmedizinische Vorsorgeuntersuchungen, Hauptverband der gewerblichen Berufsgenossenschaften e.V., Bonn.

18. Schaller, K. H., Strasser, P., Woitowitz, R. \& Szadkowski, D. (1971), Z̃. Anal. Chem. 256, 123-128.

Dr. Oster, Oskar

î́. Medizinische Klinik und Poliklinik

Abt. Klinische Chemie und Laboratoriumsmedizin

Langenbeckstr. 1

D-6500 Mainz 
